\title{
Vendor question and answer panel
}

Published online: 22 July 2006

C) Springer-Verlag 2006

Steve Balter: End users need clear documentation of how your system is configured in a way that a physicist can read without having access to service passwords. How is the machine configured? What do the local applications people do that's different from the standard configuration of the factory? When we go back a year later, we know where we are at, especially when we have systems from multiple vendors in our departments.

Philips: We may have a couple of things that may be helpful here. First of all, today's systems are digital-based systems with highly flexible configurations. We have the ability to select multiple options easily through software. I believe you are addressing the human aspect of this process in terms of communication - who needs to know what was decided and what was done. Dialog among the application person, the service person and the medical physicist is required. Systems used to have a lot better written documentation. Today, companies have moved to CDs due to cost considerations. But CDs can and are duplicated.

Access to machine configurations is another matter. I know within our organization, service personnel have different levels of service keys. It speaks to the complexity of the systems, not from an operational standpoint but from a calibration standpoint, and the unbelievable programmability of these systems. We have complaints from our own service people that they would like to do something but they don't have the right level key. Hopefully that will change. Physicists are asking for access to equipment that would allow them to do acceptance testing on equipment. Hopefully that will also change.

GE: Just to clarify quickly, everybody is moving toward less paper documentation.

Steve Balter: I have on CDs and other media information from service engineers that assumes I can read your internal formats and understand what it means. There's a need for clarity. I have no desire to get into anybody's system at a level where I can change something. I need to be able to read what you have done.
Siemens: I agree with your comment. I think it is menurelated. There is a faster innovation process so there are a lot of changes and a high complexity of the system. So that's why I think we are always lagging behind with documentation.

Steve Balter: It's not a documentation issue. Some configurations come from the factory; other configurations are changed by local service and local applications. Then someone else comes in, either a specialist or another applications person, and changes it again. I need to know how you left it when you turned it over to me so a year later when I believe I have discovered changes I can verify them.

Dr. Kathy Murray: What is the current status on any fluoroscopic units that have multi-planning or reformatting 3-D capabilities? Please comment on quality and dose issues.

Siemens: Dyna CT has been available from Siemens for one year. Dyna CT is equivalent to computed tomography. You have a rotating C-arm so you can reconstruct with emphasis on soft tissue. The biggest advantage of $\mathrm{C}$-arm systems is improved resolution, even higher than conventional CT systems. The dose for Dyna CT is comparable to standard CT machine doses.

Dr. Murray: So, are the 3-D images generated in the OR comparable to standard CT images? I've heard that they are lower in quality, but I've not actually seen them. When you use this 3-D CT in the OR for a routine case, do you get equivalent image quality?

Siemens: Yes, it's just an additional option when the machine is purchased.

Dr. Murray: And when you do that is there any increased dose if you are just getting fluoroscopic images in the OR?

Siemens: Yes, you are using the same programs.

Philips: Dr. Racadio said that he had been involved in a study at Duke looking at the amount of dose between 3-D RA (rotational angiography) and general angiography on interventional equipment. The 3-D RA concept for Philips 
started at the University of Colorado in adult coronary patients. If you looked at using 3-D RA in a coronary application compared to the typical procedure, radiation dose was reduced overall along with a reduction in contrast media. This level is probably not possible on a mobile C-arm.

GE: I know GE is working on this type of technology, but I believe Siemens had the first product on the market.

Philips: Like GE, this capability is still a work in progress on mobile C-arms. As far as CT angiography is concerned, that system is called Expert CT. Dr. Racadio showed some images of that yesterday. I don't think there's a huge dose saving doing that. There's the convenience of being able to do a CT during a difficult interventional procedure without having to stop the procedure, send the patient to CT and possibly bring them back. But as far as dose is concerned, it's probably in most cases a trade-off.

GE: I do not think that the addition of the 3-D capability to the mobile $\mathrm{C}$-arm is going to affect the basic dose operating levels of the unit in its normal 2-D mode. The mobile C-arm systems do not have sufficient power capability, so dose would be lower than doing 3-D on a fixed system, but image quality would also be poorer. If the application is for orthopedic purposes with less imagequality demands, the mobile $\mathrm{C}$-arm might meet your needs.

Tony Seibert: This is to all three panelists. I concur with Steve's comments earlier about the ability to get into the system. Let's assume I'm a technologist in a really remote location. I didn't hear any mention of quality control or a quality control phantom or automatic evaluation of the system remotely. Could each of you comment on what you have in terms of quality control and how you can verify your system is functioning properly each day? Is there a single-shot phantom? I know there's a big push for this for CR and DR outside of fluoroscopy and real-time imaging. Can you discuss some of these options?

GE: This kind of quality assurance exists at a couple of different levels. We have a phantom, the image-quality signature test phantom, which is used routinely and periodically by the field service engineers to test from the detector through the imaging chain to the display. For the rest of the system, there are phantoms that will check out the dose levels and things like resolution with real geometric magnification. For interested medical physicists, we can show you how some of this stuff works and also explain typical results. If the system has a broadband connection, we have the ability to do remote access into the system to look at system event logs that show what has been occurring. We look for patterns that might indicate that the X-ray tube is getting old, such as voltage instability. This allows the field service engineer to plan for replacing an X-ray tube or at least notify administration of this potential need in the near future - a crisis is converted into a planned event.

Philips: If this type of program needed to be set up, somebody like me would help set up and monitor the program. The service engineers have a number of different types of phantoms they use. The majority of my testing involves the SCA\&I/NEMA phantom designed to evaluate clinical conditions. Philips' remote service network (RSN) is evolving. We, too, can look at the logs on the system if the customer can provide broadband network interfaces. Today we are bringing that ability into daily application for all of our cardiac and vascular systems.

Siemens: On the Siemens system, you have two types of phantoms. One highly advanced phantom used by consultants evaluates imaging of moving targets. For the service technician, you have a standard static phantom. This checks all things like dose settings, image-quality resolution, contrast and so on. You can check in about one hour the complete system and see if there is anything going on that is not normal. We also have a remote monitoring connection to monitor event error messages. There we can see if the system doesn't work fine. This is also an ongoing process. The target of this is a warning system that allows us to identify and eliminate problems before they become a crisis.

Tony Siebert: Are any of these things extra cost or do they come as part of the system?

GE: The IQ signature test phantom ships with every system. It is part of the service engineer's kit that sits in the air-conditioned equipment room. The broadband service support comes at a charge, but I do not know the prices. IEC standards for quality assurance on X-ray equipment also define a test method including a phantom. This effort started in Europe; I am not sure if U.S. medical physicists are aware of this program. This method does not require any special service access into the system. You can verify the equipment's performance without relying on special software or tools.

Siemens: Remote service is an additional cost to the customer. I think in the long run it might be actually a cost saving because you can avoid disasters when the equipment fails because the warning signs were not seen.

Dr. Dan Young: We want to lower the dose for the pediatric patient, which is so sensitive to radiation. This group is only a fraction of the radiology community of North America, but we want to be a catalyst. Why is it so hard to get the best practice parameters for basic examinations that would cut dose? For example, for a pediatric upper GI in a 3-year-old child of standard weight, what's our frame rate, what's the $\mathrm{kV}$, what's the pulse rate, what's the copper filtration and what's the dose and why isn't that in your brochures? And why is it that your application specialists have little knowledge about pediatric applications? If we are going to make a good effort here, you need to document and distribute this information.

Siemens: It's not easy to say exactly what to do to produce good image quality for a particular patient. It's a complex problem. Manufacturers try to automate routines so the system can decide what is best in each situation. We are trying to allow the physician to concentrate on the medical procedure and not on physical parameters. That said, a lot of different customer requirements exist. So, Siemens is always interested in fulfilling the needs and wishes of customers and to adapt the system to their needs. That is why the systems are highly configurable. A single 
unique answer does not exist for every situation. You will find ten customers and ten opinions on what is best practice.

Philips: Our equipment is so programmable, but we cannot give you what you want unless we know what it is. I'm in a marketing group, but I don't market anything. I ensure that what we have works. We have a flexible system, but less than $90 \%$ of our customers ask for anything. You must tell us precisely what you would like to have for your application or your hospital. We certainly can respond.

Application training, too, often is ineffective because the hospital couldn't provide anybody to be trained while our trainers were present. The one least likely to show up for training is the physician. And I know you are very, very busy people. As pediatric radiologists and cardiologists you tend to be more knowledgeable about how systems work and are more interested in controlling dose.

We must somehow work as a team, the customer and the manufacturer, to determine the features and the requirements for systems. We need to have a dialog, and dialog requires two people - the user and the manufacturer. Get your concerns encoded properly into the machine's procedure protocols. Some type of user community group or organization is needed to work with us to optimize system configurations for pediatrics.

GE: How many people in this room have heard about the European quality criteria series of documents? There are two of them: one on general and one on pediatric radiology. Large numbers of European radiologists have put together extensive documents for different types of exams. How many of you know that these things exist? Would you agree since you know about it that this is a starting point for industry to meet your requirements as a clinician?

Keith Strauss: In support of the companies, I have had the privilege to work with them to optimize the configuration of their equipment. This takes effort. You have to jump in and do some study. All three companies have a lot of capability, but they all approach imaging concerns with different configuration schemes. So what works with one of them doesn't work with the other. One has said it can do special things, but it has to hear what you need. For you to understand what you need, you need to have a better understanding of what the equipment can do. You need someone in your institution that understands your clinical needs and can work with these companies. When was the last time you invited somebody from an imaging company to visit and observe how you work? These companies need opportunities to see what is going on in the clinic.

Dr. Young: Do you reduce the dose in only pediatric hospitals or across the board?

Philips: Across the board, but what works for you may not work for your colleague because you function differently and you have a unique set of parameters that you follow. Every manufacturer is doing everything they can to maintain good image quality at the lowest possible dose. But it's a different requirement for each type of patient or procedure. What you can do for a pediatric patient would not work well for a 350-pound adult. If a system is going into a pediatric-only application, it should be set up for those types of procedures.

Dr. Young: Let's say you work in an adult institution, but it treats adults and pediatrics. There are people there that know how to do their adult patients well, but they need some specific guidance on their pediatric patients, and they are not pediatric radiologists. Do best practice suggestions for the United States and Canada exist? Can you do that?

Philips: In some cases already companies are trying to achieve this with pediatric focus groups. Dose to the patient is an interesting thing. This dose is connected to image quality. Every manufacturer is trying to figure out how to make a better image with more perceived noise because the dose is low. The patient dose cannot be determined until we understand your image-quality requirements. In the vast majority of hospital visits as a vendor you never see a physician except when they are doing a procedure-the physician is not accessible. I think what you want is available, but you need to help us determine what that is.

Sally Mitchell: Does Philips have any plans for a triple focal spot X-ray tube on its flat panel?

Philips: I don't believe we have a triple focal spot tube. There are so many tubes in our organization that I don't know them all by heart.

Sally Mitchell: My other question is about fluoro fade. We love it because it requires no extra radiation, no extra contrast, and provides great resolution. What about flatpanel fluoro fade?

Siemens: The Siemens system can do that.

Sally Mitchell: What about Philips and GE?

Philips: Yes, it's possible. It also can be added to existing equipment in the field that is only 1 year old.

GE: Yes, we can do that. 